\title{
CREATING AUTHENTIC LEARNING ACTIVITIES IN PHARMACEUTICAL INSTRUMENTAL ANALYSIS: USING THE INTEGRATED LABORATORY NETWORK FOR REMOTE ACCESS TO SCIENTIFIC INSTRUMENTATION
}

\author{
Devon A. Cancilla \\ Scientific Technical Services \\ Western Washington University \\ Simon P. Albon \\ Faculty of Pharmaceutical Sciences \\ The University of British Columbia
}

\begin{abstract}
The Western Washington University Integrated Laboratory Network (ILN) is an initiative to provide anytime/anyplace access to scientific instrumentation for use in the classroom, laboratory, and research environments. The ILN provides students with greater opportunities to design and conduct real experiments remotely using advanced analytical instrumentation. This paper describes the use of the ILN to provide pharmaceutical sciences students at the University of British Columbia with remote access to instrumentation located at Western Washington University for the purpose of measuring metals in traditional herbal medicines. Prior to the introduction of the ILN, this type of activity would have been difficult, if not impossible, to conduct. Student feedback related to the use of the ILN was positive and supports the further development of curricular materials related to the use of remote instrumentation.
\end{abstract}

\section{KEYWORDS}

Online Laboratories, Remote Instrumentation, Chemical Measurements, Pharmaceutical Analysis, Traditional Herbal Medicines

\section{INTRODUCTION}

The Western Washington University (WWU) Integrated Laboratory Network (ILN) is an initiative to integrate scientific instrumentation and supporting instructional material into classroom, laboratory, and research environments through the use of web-based technologies. The objective of the ILN is to provide anytime/anyplace access to modern scientific instrumentation and associated expertise, with the goal of providing additional opportunities for students to engage in "mindful" scientific activities at all levels of the curriculum [1, 2]. To help understand how these goals and objectives are achieved, we have defined remote access to scientific instrumentation and expertise based on ILN access levels and the roles of an ILN Host and ILN Guest during an ILN interaction (Table 1). An ILN Host is the institution (or individual) that provides the remote access to scientific instrumentation and associated expertise, while 
the ILN Guest is the institution (or individual) that requests remote access to the instrumentation and expertise. The extent of interaction between the host and guest defines one of three ILN access levels. At each access level, instrumentation and expertise can be incorporated into classrooms, laboratories and research environments, with either the ILN Host or ILN Guest assuming the leadership or facilitation role during the interaction. For example, at ILN Level 1, the ILN Host may lead a remote lecture describing the principles and practices associated with the use of a particular instrument and then operate the instrument as a demonstration. At ILN Level 2, the ILN Guest might lead the lecture while the ILN Host may only demonstrate the operation of an instrument and act as a reference person during class discussion. ILN Level 3 might allow the ILN Guest to lead a session almost independently of the ILN Host. It is the dynamics and the level of flexibility possible in an ILN interaction and centered on the use of instrumentation that underpins the potential of the ILN for developing new teaching and learning activities to enhance science education.

This paper describes the use of the ILN in a joint project between WWU and the University of British Columbia (UBC) in the development of a new laboratory exercise allowing UBC pharmacy students to analyze traditional Chinese herbal medicines for the presence of metals using the WWU flame atomic absorption spectrophotometer (FAAS).

Table 1. ILN Access Levels and the Roles of the ILN Host and ILN Guest

\begin{tabular}{|c|c|c|c|}
\hline \multirow{4}{*}{$\begin{array}{l}\text { Increasing } \\
\text { Interactivity with } \\
\text { ILN Host }\end{array}$} & & ILN Host & ILN Guest \\
\hline & $\begin{array}{l}\text { ILN Access } \\
\text { Level } 1\end{array}$ & $\begin{array}{l}\text { Leads remote } \\
\text { lectures, demos, } \\
\text { and runs lab }\end{array}$ & $\begin{array}{l}\text { Facilitates } \\
\text { interactions, } \\
\text { limited direct } \\
\text { involvement }\end{array}$ \\
\hline & $\begin{array}{l}\text { ILN Access } \\
\text { Level } 2\end{array}$ & $\begin{array}{l}\text { Provides } \\
\text { technical } \\
\text { support during } \\
\text { lecture/demos, } \\
\text { runs instruments } \\
\text { in lab, external } \\
\text { expertise } \\
\text { provided as } \\
\text { needed }\end{array}$ & $\begin{array}{l}\text { Leads lectures } \\
\text { incorporating } \\
\text { remote access to } \\
\text { instruments, } \\
\text { facilitates lab } \\
\text { demos/use, calls } \\
\text { on external } \\
\text { expertise as } \\
\text { necessary }\end{array}$ \\
\hline & $\begin{array}{l}\text { ILN Access } \\
\text { Level } 3\end{array}$ & $\begin{array}{l}\text { Ensures } \\
\text { instruments are } \\
\text { ready, facilitates } \\
\text { interactions as } \\
\text { necessary, } \\
\text { limited } \\
\text { involvement }\end{array}$ & $\begin{array}{l}\text { Leads lectures, } \\
\text { demos, and runs } \\
\text { lab using remote } \\
\text { instrumentation, } \\
\text { requires minimal } \\
\text { participation } \\
\text { from ILN Host }\end{array}$ \\
\hline
\end{tabular}

\section{USING THE INTEGRATED LABORATORY NETWORK}

\section{A. Setting the Stage}

A number of recent studies have demonstrated that traditional herbal medicines can be contaminated with significantly high levels of heavy metals such as mercury, arsenic, and chromium [3, 4]. As the popularity and use of these alternative medicines, which are not fully regulated in the United States and Canada, has increased, it is increasingly important for practicing pharmacists to be aware of the possibility that herbal 
medicines may be contaminated with potentially harmful levels of metals [5]. This growing public health issue provides a unique opportunity for introducing pharmacy students to the analytical techniques and instrumentation used to detect the presence of metals in traditional herbal medicines. To accomplish this, the ILN was used to provide pharmacy students at UBC with remote access to the WWU FAAS system, the instrumentation capable of performing this type of analysis. Access to the FAAS provided UBC students with the unique opportunity to design and conduct field studies related to the detection of metals in herbal medicines collected in the Vancouver area. Prior to the introduction of the ILN, this laboratory exercise would not have been possible, due to the lack of an FAAS instrument for teaching purposes in the pharmacy program at UBC. Analysis of assessment data collected from students regarding the use of the ILN, through surveys, focus groups, and one-minute papers, indicates that their ILN experiences were positive. The data supports the use of the ILN as a teaching tool as well as the further development of curricular materials related to the use of remote instrumentation.

\section{B. The ILN Technology Requirements}

The ILN experience is facilitated through NetMeeting [6], a free desktop sharing program within the Microsoft Windows operating system that supports audio and video exchange as well as synchronous chat. In a shared NetMeeting session, the computer controlling the scientific instrument acts as the ILN Host computer, with the remote computer acting as the ILN Guest. Direct connection between the guest and host computers is initiated through NetMeeting at the request of the guest by "calling" the Internet Protocol (IP) address of the host computer. Once connected, the guest computer "sees" the instrument software interface on the desktop of the host computer and can request full instrument control just as if it were connected directly to the instrument. In addition, the NetMeeting window, which remains open on both the guest and host computers during a shared session, facilitates the two-way audio and video exchange. Inexpensive cameras and microphones allow the guest to "see" and "hear" the instrument and laboratory surroundings during an experiment and communicate directly with the operator at the host end while the host can see, hear and interact simultaneously with session guests. While the quality of the ILN interaction can be improved with high-end video conferencing equipment, one of the guiding principles of ILN development was affordability to maximize the learning opportunities across secondary and postsecondary educational institutions.

\section{The Pharmacy Course}

The Introduction to Pharmaceutical Analysis course at UBC (Pharmacy 325) is a required course taken by all third year pharmacy students (enrollment: 140). The course includes four hours of lecture and a three-hour laboratory period per week, respectively, over one academic term (12 weeks). The objectives of the course are to provide pharmacy students with a background in the analytical techniques used in modern pharmaceutical analysis and to engage them in context-specific analytical experiments that are relevant to their practice as pharmacists [7]. As is typical with most lecture/laboratory courses, the lecture component of Pharmacy 325 provides pharmaceutical analysis theory while the laboratory aspect of the course provides an opportunity for students to apply theoretical concepts in a "hands-on" experimental context using the associated instruments or techniques. The introduction of the new FAAS laboratory exercise and instrumental technique into the course, including remote access to the WWU instrument, required re-designing the traditional lecture/laboratory course to integrate the web-technologies and the ILN into the course format. To help students adjust to these new learning opportunities and teaching methods, the WWU faculty member (the ILN Host) was introduced online, as a "Virtual Professor" during the opening lecture of the course using a remote Internet connection and then appeared periodically as a course guest throughout the term. The initial introduction was important both to troubleshoot connectivity issues and to provide many of the students with their first remote classroom experience. NetMeeting along with the web cameras and microphones allowed two-way audio/video 
exchange between the WWU faculty and UBC students and faculty member at all times during the introductory session as well as subsequent guest appearances. To support the new laboratory activities, later in the course the WWU faculty member traveled to the UBC campus to present a "live" lecture on the principles of atomic absorption spectroscopy as well as a remote demonstration of the FAAS system. (Figure 1). The UBC campus is approximately 70 miles north of the WWU campus, across the US/Canada border. This live lecture presentation provided students with an example of how the device would be used during the laboratory, presented students with the opportunity to interact with the WWU faculty member prior to conducting the remote experiments and helped capture the excitement and uniqueness of the ILN learning opportunity for students in the course.

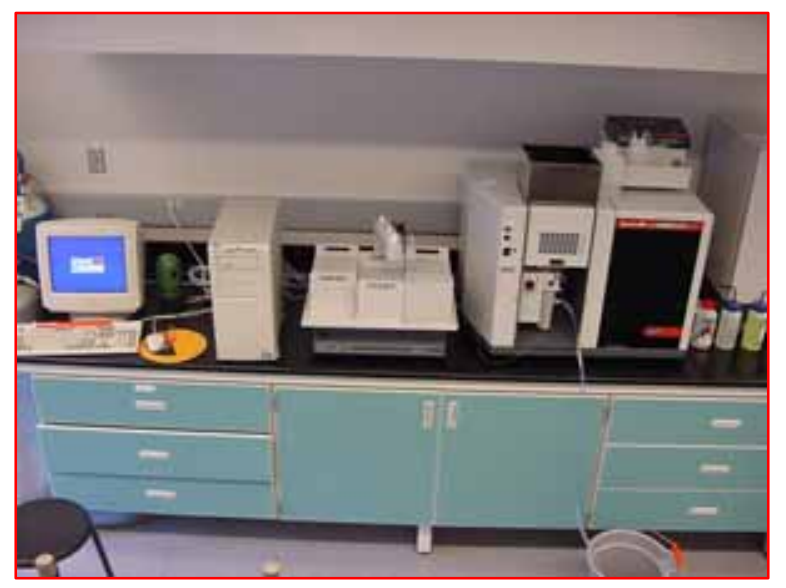

Figure 1. The WWU FAAS System Used Remotely by Pharmacy 325 Students in Lecture and Laboratory Components of the Course

The principles of FAAS and the application of FAAS to the analysis of metals in herbal medicines were presented by connecting to the WWU FAAS system during a live introductory lecture and in the course laboratory. $71 \%$ of the students agreed "significantly" or "very significantly" that seeing the AAS instrument in lecture before using it in the laboratory was valuable.

\section{The Laboratory}

Two three-hour laboratory periods were dedicated to students' investigation into the presence of metals in traditional herbal medicines. A virtual laboratory was set up within the UBC pharmaceutical analysis teaching laboratory to allow laboratory groups (three to four students per group) to remotely connect to the WWU FAAS and to interact with WWU faculty and staff (Figure 2).

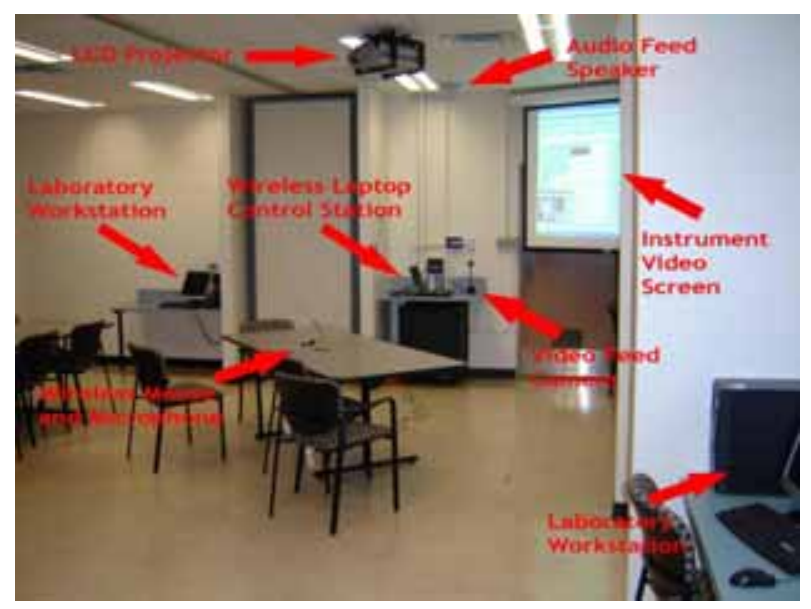
Figure 2. The UBC Virtual Laboratory Connecting to the
WWU FAAS System

The UBC laboratory was set-up to include a virtual laboratory, allowing the WWU FAAS to be operated in real time by the small student laboratory groups. The virtual laboratory included audio/video connections to WWU and the software necessary to control the WWU FAAS. In addition, an IP printer was incorporated into the UBC Virtual Laboratory to print analytical reports from the WWU FAAS directly to the UBC teaching laboratory.

Each laboratory group of students collected traditional Chinese herbal medicines from pharmacies in the Vancouver area prior to the start of the first lab period. During the first lab period, each group completed an initial experiment remotely using the WWU FAAS system to determine the experimental conditions under which the actual analysis of their herbal samples would be conducted. What the students saw 
during this step of the experiment is illustrated in Figure 3. NetMeeting, web cameras and microphones again facilitated this interaction between UBC students and WWU faculty and technicians.

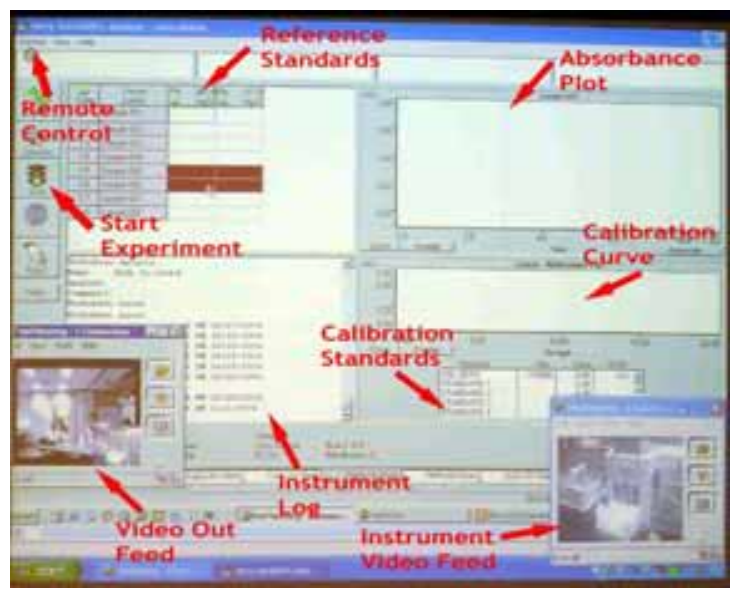

Figure 3. Operating the WWU FAAS Remotely: What the Student Sees

Students operated the WWU FAAS system remotely in real time over the Internet, generating the experimental data needed for the analysis of heavy metals in their herbal samples. Audio/video connections allowed the FAAS to be seen in operation and to let UBC and WWU participants see and talk to each other during the experiment. Completed data sets were printed in the UBC Virtual Lab and were posted on the Pharmacy 325 website.

In addition, following techniques described in the literature [5], students and teaching assistants prepared the traditional herbal medicines for analysis by FAAS during the first laboratory period (Figure 4). These samples were then packaged and sent via overnight courier to the WWU analytical laboratory. During the second laboratory, samples were loaded onto the WWU FAAS instrument's autosampler, a robotic device was used to automatically introduce samples into the instrument, and each UBC group had the opportunity to use the ILN to remotely operate the FAAS and to interact with the WWU faculty and staff. Data from all of the lab groups were pooled at the end of each laboratory so that the results from all of the classes could be evaluated.

Figure 4. Laboratory Activities for the Preparation of Traditional Herbal Medicines

Students were provided with a tutorial on the procedures of extracting metals from herbal medicines. They then prepared their samples in the UBC laboratory and sent them to WWU for analysis by FAAS.

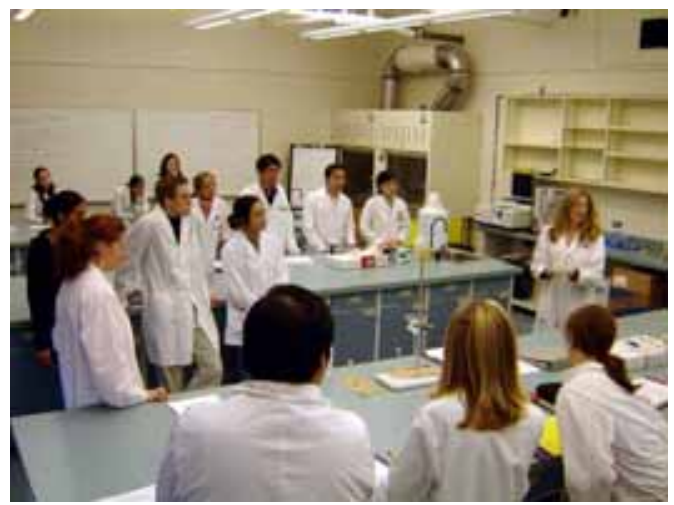

\section{E. The Results: Student Perceptions}

During their investigations of the presence of metals in traditional herbal medicine, UBC students had a number of opportunities to interact with faculty and staff at WWU. These interactions occurred both in person and remotely, through the ILN. Of the 128 students responding to a survey following completion of the exercise (response rate: $92 \%$ ), $78.1 \%$ of the students rated the overall learning activity (remote access to the WWU FAAS for the analysis of metals in traditional medicines) as either "good" or "very good". When asked how they would rate the use of the ILN as a teaching tool in the lecture component of the course, $83 \%$ of student respondents gave the experience a rating of "good" or "very good". When asked whether the ILN should continue to be developed as a teaching tool, 83.6\% of the students either "agreed" or "strongly agreed" with this statement. Additional survey elements, such as focus group 
comments and one-minute papers, provided positive support for the use of the ILN as a teaching tool in the pharmaceutical analysis course.

\section{SUMMARY AND CONCLUSIONS}

The WWU ILN was successfully used to provide new teaching and learning opportunities within the pharmacy program at UBC. By remotely accessing instrumentation located at WWU, UBC students had the opportunity to support their learning in the lecture component of the course and conduct a full laboratory exercise on the analysis of metals in traditional herbal medicines. The lecture and laboratory learning opportunities provided by the ILN were relevant to their training as pharmacy practitioners and were previously impossible to conduct due to the lack of an FAAS system for teaching purposes in the pharmaceutical analysis course. In addition, the ILN provided UBC students with an opportunity to interact and collaborate with faculty and staff from WWU in developing the procedures and protocols necessary to complete this experiment. The ability of students to collaborate with personnel from other institutions is an important element of the ILN and one rarely provided to students in a traditional laboratory environment.

Lack of access to scientific instrumentation has often been cited as a major reason that teaching laboratories have become "cookbook" exercises with little to do with teaching how science is actually conducted. The ILN has provided a clear example of how analytical lecture/laboratory courses can be modified to use advanced scientific instrumentation remotely to overcome these obstacles and revamp the way in which laboratory-based sciences can be taught.

\section{REFERENCES}

1. Cancilla, D. A. Initial Design and Development of an Integrated Laboratory Network (ILN): A New Model for the Use of Instrumentation in the Undergraduate Curriculum. Journal of Chemical Education 81 (12): 1809-1813, 2004.

2. Cancilla, D. A. Integrated Laboratory Network: Better Access to Scientific Instrumentation. The Sloan Consortium, 2004. Online: http://www.sloan-c.org/effective/details2.asp?ACC_ID=59.

3. Ernst, E. Toxic heavy metals and undeclared drugs in Asian herbal medicines. Trends in Pharmacological Sciences 23 (3): 136-139, 2002.

4. Au, A. M., R. Ko, F. O. Boo, R. Hsu, G. Perez, and Z. I. Yang. Screening methods for drugs and heavy metals in Chinese patent medicines. Bulletin of Environmental Contamination and Toxicology 65 (1): 112-119, 2000.

5. Eisenberg, D. M., R. B. Davis, S. L. Ettner, S. Appel, S. Wilkey, M. Van Rompay, and R. C. Kessler. Trends in alternative medicine use in the United States, 1990-1997. The Journal of the American Medical Association 280 (18): 1569-1575, 1998.

6. NetMeeting Home Page. Available at: http://www.microsoft.com/windows/netmeeting/. Accessed April 21, 2005.

7. Albon, S. P., and H. Hubball. A Learning-Centered Course in Pharmaceutical Analysis. American Journal of Pharmacy Education 68 (5): article 114, 2004.

\section{ACKNOWLEDGEMENTS}

This project was funded in part by a grant from the National Science Foundation's Course, Curriculum, and Laboratory Improvement Proof-of-Concept program (Grant \# 0341019). Support from UBC's Office of Learning Technology and Teaching and Learning Enhancement Fund is gratefully acknowledged. 


\section{ABOUT THE AUTHORS}

Devon A. Cancilla, Western Washington University, is the Director of Scientific Technical Services and an associate professor of environmental science. He has been the lead investigator in the development of the Integrated Laboratory Network, a project currently funded by the National Science Foundation. His most recent article on the ILN, Initial Design and Development of an Integrated Laboratory Network (ILN): A New Model for the Use of Instrumentation in the Undergraduate Curriculum, was published by the Journal of Chemical Education [J. Chem. Ed., 81 (12):1809, 2004.].

Simon P. Albon, University of British Columbia, is a Senior Instructor in the Division of Biomolecular and Pharmaceutical Chemistry at the Faculty of Pharmaceutical Science. He currently teaches a third-year lecture/laboratory course in pharmaceutical analysis and has research interests in scholarly teaching and the scholarship of teaching and learning with a focus on learning-centered education in the basic pharmaceutical sciences. Simon is currently involved in an NSF funded pilot program to evaluate the ILN for implementation at UBC. His most recent article, A Learning-Centered Course in Pharmaceutical Analysis, was published by the American Journal of Pharmaceutical Education [Am J Pharm Ed. 68 (5): article 114, 2004.]. 